\title{
POTENTIAL FOR APPLYING CONTINUOUS DIRECTED EVOLUTION TO PLANT ENZYMES
}

Jorge D. García-García ${ }^{1, \star}$, Jaya Joshi ${ }^{1, \star}$, Jenelle A. Patterson ${ }^{1}$, Lidimarie Trujillo-Rodriguez ${ }^{2}$, Christopher R. Reisch ${ }^{2}$, Alex A. Javanpour ${ }^{3}$, Chang C. Liu ${ }^{3,4,5}$, and Andrew D. Hanson ${ }^{1}$

${ }^{1}$ Horticultural Sciences Department, University of Florida, Gainesville, FL, USA

${ }^{2}$ Department of Microbiology and Cell Science, University of Florida, Gainesville, FL, USA

${ }^{3}$ Department of Biomedical Engineering, University of California, Irvine, Irvine, CA, USA

${ }^{4}$ Department of Chemistry, University of California, Irvine, Irvine, CA, USA

${ }^{5}$ Department of Molecular Biology and Biochemistry, University of California, Irvine, Irvine, CA, USA

*These authors contributed equally to this work

Correspondence to: jgarciagarcia@ufl.edu; jjoshi@ufl.edu; patterson.j@ufl.edu

\section{SUMMARY}

Plant evolution has produced enzymes that may not be optimal for maximizing yield and quality in today's agricultural environments and plant biotechnology applications. By improving enzyme performance, it should be possible to alleviate constraints on yield and quality currently imposed by kinetic properties or enzyme instability. Enzymes can be optimized faster than naturally possible by applying directed evolution, which entails mutating a target gene in vitro and screening or selecting the mutated gene products for the desired characteristics. Continuous directed evolution is a more efficient and scalable version that accomplishes the mutagenesis and selection steps simultaneously in vivo via error-prone replication of the target gene and coupling of the host cell's growth rate to the target gene's function. However, published continuous systems require custom plasmid assembly, and convenient multipurpose platforms are not available. We discuss two systems suitable for continuous directed evolution of enzymes, OrthoRep in Saccharomyces cerevisiae and EvolvR in Escherichia coli, and our pilot efforts to adapt each system for high-throughput plant enzyme engineering. To test our modified systems, we used the thiamin synthesis enzyme THI4, previously identified as a prime candidate for improvement. Our adapted OrthoRep system shows promise for efficient plant enzyme engineering.

Key words: Protein engineering; Synthetic biology; Linear plasmids; Error-prone polymerases; CRISPR/Cas9; Directed evolution 


\section{INTRODUCTION}

Enzyme evolution is naturally driven by beneficial mutations that are selected over many generations, but this inherently slow and random process does not necessarily produce optimally adapted enzymes, especially for new environments or applications [1,2]. In fact, many enzymes - in plants as in other organisms - are merely 'good enough' to support metabolism. For example, enzymes with sub-par kinetic properties can limit key metabolic pathways, and the frequent replacement of short-lived enzymes can divert resources from biomass synthesis and other productive uses $[2,3]$. There is thus much potential for plant improvement by reducing enzyme inefficiencies [4,5], a goal that can be advanced by applying approaches from outside plant biology.

Synthetic biology (SynBio) offers a suite of such approaches. SynBio lies at the intersection between discovery-driven biology and goal-oriented engineering and brings a fresh perspective to plant improvement by adding to the breeding toolbox [6,7]. SynBio has enormous potential in enzyme engineering, ranging from improving existing catalytic activities to creating new ones [7-9]. Early enzyme engineering in the 1980s focused on rational (re)design by site-specific mutagenesis [10]. However, evolving enzymes through semi-rational mutagenesis depends on knowledge of structurefunction relationships that is often unavailable [11] and is time- and labor-intensive, particularly when multiple mutations are needed to achieve the desired improvement. A classic example of the challenges of enzyme improvement by site-specific mutation is Rubisco, ribulose-1,5-bisphosphate carboxylase/oxygenase [12]. Attempts to improve Rubisco's carboxylase activity in plants have been complicated by the number of possible target residues, with roles ranging from conformational flexibility [13] to protein-protein interactions $[14,15]$ to effector/inhibitor binding $[16,17]$. It is therefore close to impossible to predict an optimal set of mutations to maximize catalytic efficiency.

\section{Directed Evolution}

An alternative to rational design approaches is directed evolution [18], which takes a cue from nature by harnessing the power of random mutagenesis. Directed evolution typically begins by diversifying a target gene by introducing random mutations to generate large libraries of mutated sequences. The genes encoded in these mutated sequences are then expressed, and the enzymes screened in vitro or in vivo for the desired properties [19]. The advantage of directed evolution is that its outcomes are not constrained by knowledge about the target enzyme. Rather, it is an 'act first, ask questions later' approach in which mutations are made iteratively or combinatorially in the target gene until the desired outcome is obtained.

The effects of cumulative mutations on enzyme activity can be visualized as a 'fitness landscape' in which compounding beneficial changes lead to fitness peaks and detrimental changes cause fitness 
valleys [20] (Figure 1). Given the existence of fitness valleys, reaching an optimal peak may require 'valley crossing' through intermediate steps that are deleterious [21,22]. Traversing valleys can occur by relaxing selective pressure or by introducing multiple mutations simultaneously, effectively skipping over the intermediate, less-fit steps. Directed evolution can cope with fitness valleys by introducing multiple mutations during each round of mutagenesis, but its separate screening step is labor-intensive, which limits throughput capacity. Tuning selection pressure is likewise laborintensive.

\section{Continuous Directed Evolution}

Continuous directed evolution is a 'next-generation' version of directed evolution that accomplishes the mutagenesis and screening steps simultaneously by continuously applying selection pressure throughout the mutation process [23-25]. In continuous directed evolution, the function of the target gene is typically coupled to the growth rate of the host microbe by forcing the microbe to rely on the target gene, e.g. by complementing the loss of an essential host activity with a plasmid-borne target gene. Using this method, improved target genes are immediately identifiable with no need for additional screening [26-28]. Also, tracking enzyme function in vivo avoids possible artifacts of in vitro screening assays, which may be poor facsimiles of physiological conditions. A particular advantage of continuous directed evolution is its scalability, which allows exploration of many trajectories through a fitness landscape simultaneously (which is challenging with classical directed evolution, see above). Some continuous directed evolution platforms are also compatible with automation, which furthers scalability and industrialization [29].

The first successful continuous directed evolution system for proteins was Phage-Assisted Continuous Evolution (PACE), in which M13 bacteriophage carry a plasmid-borne target gene that is iteratively propagated and mutated within infected $E$. coli host cells [23]. The target gene's function is coupled to the induction of an essential bacteriophage virulence factor, plll, such that more active target gene variants result in increased phage infectivity and propagation. Although novel plll induction mechanisms are being developed to adapt PACE for broader applications [25], the utility of PACE for metabolic enzyme evolution remains limited, there being no generalizable way to link plII induction to enzyme traits such as kinetic characteristics or half-life.

Fortunately, continuous directed evolution systems suitable for metabolic enzyme improvement have now been introduced. Below, we outline the Saccharomyces cerevisiae OrthoRep and Escherichia coli EvolvR systems; each has unique and innovative features that avoid some of the limitations of PACE. 


\section{OrthoRep}

The yeast OrthoRep system was developed from the p1 and p2 linear cytoplasmic plasmids of Kluyveromyces lactis [26,30-32]. The p1-specific DNA polymerase TP-DNAP1 was transferred from $\mathrm{p} 1$ to a nuclear plasmid, leaving a backbone into which a target gene can be introduced (Figure 2A); p2 remains in its native state, encoding its own DNA polymerase (TP-DNAP2) and the machinery needed to transcribe both $\mathrm{p} 1$ and $\mathrm{p} 2$ [32]. For continuous directed evolution, error-prone versions of TP-DNAP1 have been engineered; these polymerases introduce mutations into the p1-borne target gene without increasing the genomic mutation rate (Figure 2A). Error-prone TP-DNAP1s have mutation rates as high as $1 \times 10^{-5}$ substitutions per base, which is up to $10^{5}$ times that of the host genome [26,32]. The $\sim 10^{5}$-fold mutational acceleration, combined with serial passaging, enables rapid, continuous evolution of the target gene entirely in vivo. OrthoRep is compatible with highthroughput, automated systems, including some that can impose diverse and adjustable selection pressures to help cross fitness valleys [29].

\section{EvolvR}

The E. coli EvolvR system uses a fidelity-reduced variant of E. coli DNA polymerase I (Poll3M or Poll5M) fused to a CRISPR-guided nickase, nCas9 (derived from Streptococcus pyogenes Cas9), which directs the polymerase to a specific location on the target gene [27] (Figure 2B). EvolvR is a two-plasmid system in which one plasmid ( $\mathrm{pEv}$ olvR) harbors the Poll-nCas9 fusion plus a singleguide RNA (sgRNA) and the other plasmid (pTarget) carries the target gene. The EvolvR system offers a targeted mutation rate of $\sim 10^{-5}$ to $10^{-6}$ mutations per nucleotide per generation averaged over a 56-bp window, with the highest rates closest to the nick site [27].

\section{Limitations of Continuous Directed Evolution Systems}

The application of OrthoRep, EvolvR, or other continuous directed evolution systems to highthroughput improvement of enzymes is subject to certain (mostly obvious) constraints, as follows. (i) As noted above, the target enzyme typically needs to be essential to the growth of the platform cells so that the growth rate depends on the enzyme's activity. (ii) The target enzyme must be expressed mainly in soluble form to avoid selecting for mutations that merely increase solubility in the alien platform cell milieu instead of improving the desired characteristic immediately. (iii) To maximize applicability, the system should function in minimal or defined growth media. While OrthoRep was developed using defined media, EvolvR was developed using rich medium and its compatibility with minimal media was not explored.

To date, OrthoRep and EvolvR have only been used to evolve microbial enzymes, and for just two types of applications (antibiotic resistance and thermal adaptation) [27,29,32]. Furthermore, 
particularly for OrthoRep, the target gene plasmid construct is tailor-made and cannot be easily repurposed for different genes of interest.

The aim of this work was two-fold: (i) to adapt OrthoRep to facilitate simple, high-throughput cloning of target genes; and (ii) to establish the potential of OrthoRep and EvolvR to improve the characteristics of metabolic enzymes, which - for EvolvR - required use of minimal medium. We chose the thiamin thiazole synthesis enzyme THI4 as the target for these proof-of-concept studies. Plant and yeast THI4s are suicide enzymes that self-inactivate after a single catalytic cycle because they obtain the sulfur atom needed to form the thiazole product by destroying an active-site cysteine residue. Such THI4s are consequently energetically expensive to operate [3,33-35]. In contrast, certain prokaryotic THI4s are truly catalytic, i.e. perform multiple reaction cycles; these enzymes use sulfide as sulfur source [36,37]. Thermovibrio ammonificans THI4 (TaTHI4) belongs to this group $[35,38]$. However, like other characterized prokaryotic THI4s, TaTHI4 prefers anaerobic, high-sulfide conditions, making it ill-suited for function in plant cells. Our long-term goal is therefore to evolve the non-suicidal TaTHI4 to function efficiently in plant-like conditions (aerobic, low sulfide). To explore the feasibility of this goal we adapted OrthoRep and EvolvR systems to complement thiamin auxotrophic yeast or E. coli strains with native yeast THI4 or TaTHI4, respectively.

\section{MATERIALS AND METHODS}

\section{Chemicals, enzymes, and oligonucleotides}

Chemicals were purchased from Sigma-Aldrich (St. Louis, MO) unless otherwise stated. Phusion® High-Fidelity DNA polymerase and restriction enzymes (New England Biolabs, Ipswich, MA) were used for PCR reactions and gene cloning. Oligonucleotide primers were obtained from Eurofins Genomics LLC (Louisville, KY) and are listed in Supplementary Table S1. All constructs were sequence-verified.

\section{OrthoRep}

Construction of multipurpose $p 1$ integration vector.

In previous work, target gene expression in yeast was enhanced by placing a poly-A tail/selfcleaving ribozyme (RZ) sequence downstream of the target gene [39]. We therefore built a multipurpose p1 integration vector from 172-YTK-P4 (containing the poly-A/RZ self-cleaving ribozyme cassette) and GR306 (used as backbone, with the fluorescent reporter mKate2 in its cloning region), as shown in Figure 3. We excised the poly-A/RZ module from 172-YTK-P4 using $\mathrm{BspHI}$ and EcoRI, blunting the product with T4 polymerase (New England Biolabs). A Smal site was introduced into GR306 after the Sphl site (Figure 3). GR306 was then digested with Smal, dephosphorylated, and ligated with the blunted poly-A/RZ module, whose correct orientation was 
confirmed. The resulting plasmid is a multipurpose $\mathrm{p} 1$ integration vector in which $m$ Kate2 can be replaced with any target gene. The yeast THI4 coding sequence (ScTHI4) was synthesized by GenScript (Piscataway, NJ), and PCR amplified using primers 1 and 2, adding Nsil and Sphl sites to the $5^{\prime}$ and $3^{\prime}$ ends, respectively. The amplicon was introduced into pGEM T-Easy by TA cloning (Promega) using the manufacturer's protocol. ScTHI4_pGEM (15 $\mu \mathrm{g})$ and multi-purpose p1 integration vector $(15 \mu \mathrm{g})$ were digested with Nsil and Sphl, gel-purified, cleaned using a GeneJET Kit (Thermo Scientific), and ligated to give the plasmid p1_ScTHI4 integration vector. In parallel, the multi-purpose $\mathrm{p} 1$ integration vector was digested with Nsil and Sphl to release mKate2, blunted, and self-ligated to generate $\mathrm{p} 1$ empty integration vector.

Transformation of the yeast $\triangle$ THI4 strain with wild type and error-prone polymerases.

TP-DNAP1 wild type (TP-DNAP1_wt) or error-prone (TP-DNAP1_611 and TP-DNAP1_633) polymerases were cloned into nuclear plasmids containing centromere and autonomous replication sequences (CEN/ARS) and a HIS3 marker. The resulting plasmids were used to transform the

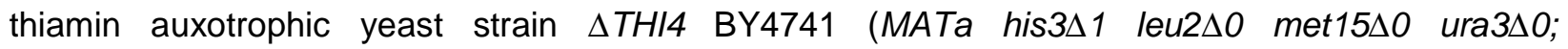
THI4A::KanMX) following the PEG-3350/Tris-EDTA/lithium acetate/salmon sperm DNA method with some modifications [40]. The transformants were plated on a synthetic, complete medium (SC; yeast nitrogen base, drop-out mix (USBiological Life Sciences, Cat No. D9540), and 2\% (w/v) glucose) minus histidine and supplemented with $100 \mathrm{nM}$ thiamin. Colonies were screened by colony PCR to confirm the presence of the respective TP-DNAP1 sequences (forward primer 3 for all cases and specific reverse primers 4 for TP-DNAP1_wt, 5 for TP-DNAP1_611 and 6 for TP-DNAP1_633).

Integration of ScTHI4 into the p1 plasmid.

The p1_ScTHI4 and p1_empty integration vectors $(10 \mu \mathrm{g})$ were linearized by digestion with Scal. After inactivation at $80 \stackrel{\circ}{ } \mathrm{C}$ for $20 \mathrm{~min}$, the digest was used to transform strain GA-Y319 (MATa can1

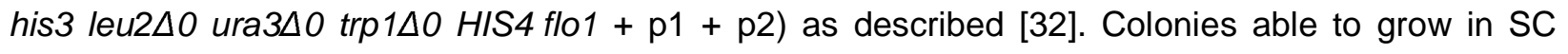
medium minus leucine were screened by agarose gel analysis of whole-cell DNA [32] for the presence of both the $\mathrm{p} 1$ and $\mathrm{p} 2$ plasmids, as well as the successfully recombined p1_ScTHI4 (Figure S1A) and p1_empty (data not shown).

\section{Protoplast fusion of $\triangle T H I 4 B Y 4741$ and GA-Y319 strains.}

Cultures of recipient ( $\triangle T H / 4$ BY4741) cells transformed with TP-DNAP1 wild type or error-prone polymerases) and donor (GA-Y319 harboring p1_ScTHI4/p2 or p1_empty/p2) strains were grown overnight in YPD medium. Both strains ( $\sim 0.3 \mathrm{~g}$ of cells) were pretreated with a solution of $\beta$ mercaptoethanol plus $60 \mathrm{mM}$ EDTA and then incubated with $6 \mathrm{U} / \mathrm{mL}$ zymolyase (Zymo Research) in 
Buffer I to remove the cell wall. The resulting protoplasts were fused in presence of polyethylene glycol-3350 (PEG-3350) and $\mathrm{Ca}^{2+}$ (Buffer II) essentially as described previously [41,42]. After incubation for $30 \mathrm{~min}$ at $30^{\circ} \mathrm{C}$, the protoplasts were pelleted by centrifugation (10 min, $700 \mathrm{~g}$ ) and gently re-suspended in Buffer III. Finally, cells were embedded in 3\% (w/v) agar (dilutions 2:1000 to 2:100) with selective medium maintained at $42{ }^{\circ} \mathrm{C}$ (SC minus leucine, histidine and tryptophan supplemented with $0.6 \mathrm{M} \mathrm{KCl}, 5 \mathrm{~g} / \mathrm{L}$ ammonium sulfate and $100 \mathrm{nM}$ thiamin), and the plates were incubated at $30^{\circ} \mathrm{C}$. Colonies were recovered from plates and replated on SC medium minus leucine, histidine and tryptophan supplemented with $1 \mathrm{~g} / \mathrm{L}$ monosodium glutamate, $100 \mathrm{nM}$ thiamin, and 200 $\mu \mathrm{g} / \mathrm{mL}$ G418. Individual colonies from plates were then used to inoculate liquid cultures of the same medium to confirm the presence of p1_ScTHI4/p2 and p1_empty/p2 by agarose gel analysis (Figure S1B). The compositions of Buffers I, II, and III are given in Table S2.

\section{Functional complementation in yeast.}

Independent clones of $\triangle T H I 4$ BY4741 carrying nuclear plasmids harboring TP-DNAP1_wt, TPDNAP1_611, or TP-DNAP1_633 and plasmids p1_ScTHI4 or p1_empty plus wild type p2 were used to inoculate $3 \mathrm{~mL}$ of $\mathrm{SC}$ minus leucine, histidine, and tryptophan supplemented with $1 \mathrm{~g} / \mathrm{L}$ monosodium glutamate, $100 \mathrm{nM}$ thiamin, and $200 \mu \mathrm{g} / \mathrm{mL}$ G418 (complementation medium). After 48 $\mathrm{h}$ of incubation at $30 \stackrel{\circ}{\circ}$ and $200 \mathrm{rpm}$, cells were pelleted $(3,000 \mathrm{~g}, 5 \mathrm{~min})$ and washed in $5 \mathrm{~mL}$ of complementation medium minus thiamin. Aliquots diluted to $\mathrm{OD}_{660 \mathrm{~nm}}=0.05$ were inoculated in complementation medium plus or minus thiamin and growth was monitored at $\mathrm{OD}_{660 \mathrm{~nm}}$.

\section{EvolvR}

Bacterial strains and plasmid construction.

The T. ammonificans THI4 (TaTHI4) gene was recoded for E. coli and synthesized by GenScript (Piscataway, NJ). The EvolvR plasmid ( $\mathrm{pEv}$. Addgene (plasmid 113077). This plasmid has a pBR322 origin of replication and a kanamycin resistance cassette. A tiling approach [43] was used to design a set of guide RNAs targeting different regions of TaTHI4, which were cloned into pEvolvR by Golden Gate assembly. Primers are listed in Supplementary Table 1. The pTarget plasmid, pTaTHI4, was a pCDF duet vector (Novagen) backbone with a pLlacO1 promoter [44] driving TaTHI4 and had a streptomycin/spectinomycin resistance marker.

\section{Media and culture conditions.}

The pEvolvR and pTaTHI4 plasmids were co-transformed into an E. coli MG1655 $\Delta$ thiG strain constructed by recombineering [38]. Three independent clones of each construct were used to inoculate 
$2 \mathrm{~mL}$ of MOPS medium [45] containing $0.2 \%$ (w/v) glycerol, $100 \mathrm{nM}$ thiamin, $50 \mu \mathrm{g} / \mathrm{mL}$ kanamycin, and $100 \mu \mathrm{g} / \mathrm{mL}$ spectinomycin. After incubation at $37^{\circ} \mathrm{C}$ for $18 \mathrm{~h}$, cells were harvested by centrifugation, washed five times with $1 \mathrm{ml}$ thiamin-free MOPS medium and resuspended in $500 \mu \mathrm{L}$ of the same medium. Liquid cultures of $5 \mathrm{~mL}$ of MOPS medium containing $0.2 \%(\mathrm{w} / \mathrm{v})$ glycerol, $1 \mathrm{mM}$ IPTG, $25 \mu \mathrm{g} / \mathrm{mL}$ kanamycin, $50 \mu \mathrm{g} / \mathrm{mL}$ spectinomycin, and $1 \mathrm{mM}$ cysteine were inoculated with washed cells to an $\mathrm{OD}_{600 \mathrm{~nm}}$ of 0.02 . Cells were grown at $37^{\circ} \mathrm{C}$ with shaking at $225 \mathrm{rpm}$; growth was monitored by measuring $\mathrm{OD}_{600 \mathrm{~nm}}$ with a $1.5 \mathrm{~cm}$ pathlength cell.

\section{RESULTS AND DISCUSSION \\ OrthoRep}

We adapted OrthoRep for high-throughput use by constructing a multi-purpose $\mathrm{p} 1$ integration vector based on the GR306 plasmid (Figure 3A), to which we added a convenient directional cloning site for target genes (with Nsil and Sphl sites) and a downstream poly-A/RZ module to enhance expression [39]. Because the poly-A/RZ module contains 75 consecutive adenosine residues it is inherently unstable and shortens with repeated propagation. When constructing the multi-purpose integration vector, we verified that $\geq 72$ adenosine residues remained. Note that this vector would need to be reconstructed if further propagation results in failure to reach this threshold.

In continuous directed evolution platforms, target gene function must be coupled to host growth rate. To evaluate the adapted OrthoRep system we therefore tested for complementation of a yeast thiazole auxotroph ( $\triangle T H I 4$ BY4741) by native ScTHI4. We produced $\triangle T H I 4$ BY4741 strains harboring the p2 plasmid and combinations of p1_empty or p1_ScTHI4 with wild type or error-prone (TP_DNAP1_611 or 633) DNA polymerases in the nuclear CEN6/ARS4 plasmid (Figure 3B). Complementation tests were first run on plates to qualitatively compare the growth of the various strains. The selective medium (minus thiamin, leucine, and histidine) also lacked tryptophan to eliminate any donor GA-Y319 cells (tryptophan auxotroph) left after protoplast fusion (Figure 3B). All strains grew in the presence of $100 \mathrm{nM}$ thiamin and the absence of leucine and histidine (Figure 4A), indicating that all three TP_DNAP1 polymerases were working in trans with the recombined p1_ScThi4. All three $\triangle T H I 4$ BY4741 strains carrying p1_ScTHI4 grew without thiamin, whereas the empty vector controls did not, as expected. However, the growth rates of the strains carrying p1_ScTHI4 differed. Cells having the wild type polymerase showed substantial growth after 4.5 days, while cells having TP_DNAP1_611 or 633 did not reach comparable growth until 7 and 8 days, respectively, likely due to the lower copy number of p1_ScTHI4 maintained by error-prone TP_DNAP1s, resulting in less expression of THI4. Of the error-prone polymerases, TP_DNAP1_611 gave better growth and so was used for further experiments in liquid medium. 
As with solid medium, all strains grew in the presence of thiamin in liquid medium, although the growth of the TP_DNAP1_611 strain was slower than that of the TP_DNAP1_wt strain (Figure 4B). The p1_ScTHI4 plasmid conferred the ability to grow without thiamin supplementation. However, cells expressing either polymerase showed a growth lag compared to the thiamin-supplemented controls, with the growth of the TP_DNAP1_611 strain delayed more than that of the TP_DNAP1_wt strain and showing more variation among the replicates (Figure 4B). As the copy number of p1_ScTHI4 is lower in strains with the mutant TP-DNAP1 variants compared to WT, expression of ScTHI4 is therefore lower and will result in relatively slower growth. Despite the growth lag of the TP_DNAP1_611 strain, the growth period remains short enough (6 days) for continuous directed evolution to be logistically feasible.

\section{EvolvR}

As described in the Introduction, we set out to evolve the non-suicidal TaTHI4 from the bacterium $T$. ammonificans to improve its activity in aerobic, low sulfide conditions similar to those in plant cells. TaTHI4 has a limited ability to complement an E. coli thiazole auxotroph under aerobic conditions when supplemental cysteine is given to increase the internal sulfide level $[38,46]$. Because the TaTHI4 coding sequence is $0.8 \mathrm{~kb}, 13$ target sgRNAs are needed to cover the whole gene to saturation. We therefore used a tiling approach and designed a set of 13 guide RNAs to target the nickase-polymerase fusion to successive regions of TaTHI4. Each pEvolvR construct contained a single guide RNA; we did not concatenate multiple guide RNAs in the same construct because this leads to plasmid instability due to repeated sequence elements. The 13 individual pEvolvR plasmids and the pTarget plasmid harboring TaTHI4 were then co-transformed into the MG1655 $\Delta$ thiG strain.

To perform directed evolution, we chose the pEvolvR plasmid containing the Poll3M DNA polymerase I variant [27]. First, we checked whether TaTHI4 expressed from the pTarget plasmid could complement the thiazole auxotrophy of the $\Delta$ thiG strain in the absence of the pEvolvR plasmid. Cell growth in thiamin-supplemented medium confirmed that TaTHI4 expression was not toxic (Figure 5A). In medium without thiamin and supplemented with cysteine, the strain carrying the TaTHI4 pTarget plasmid reached an OD 2.5-fold higher than the empty vector control, confirming the ability of TaTHI4 to complement thiazole auxotrophy (Figure $5 \mathrm{C}$ ). (The initial burst of growth followed by a plateau seen in the empty vector control is attributable to traces of thiazole that contaminate the carbon source or other medium constituents [47]. Active charcoal and ion-exchange pretreatments of the glycerol carbon source were tested in attempts to reduce this unwanted growth but had little or no effect.) In contrast, $\Delta$ thiG cells carrying the pool of pEvolvR plasmids as well as the TaTHI4 pTarget plasmid showed no growth in cysteine-supplemented medium, although these 
cells grew well with thiamin supplementation (Figure 5B, C). This result indicates that pEvolvR expression imposes an insupportable burden on cells cultured in minimal medium, unlike the situation in rich medium [27]. Reducing the copy number of pEvolvR expression level by replacing the high-copy origin (pBR322) with a lower copy variant (p15A), removing non-essential components from the pEvolvR plasmid, or replacing the $0.2 \%$ glycerol carbon source with $0.4 \%$ or $0.8 \%$ glucose did not rectify the problem. Possible future solutions include encoding a single copy of the nickasepolymerase fusion in the E. coli genome or modifying the EvolvR system for use in a more robust chassis bacterium such as Pseudomonas putida [48].

\section{FUTURE PERSPECTIVES}

The above pilot studies indicate that our adapted OrthoRep system is in principle ready for highthroughput evolution of enzymes from plants or other organisms. The EvolvR system, on the other hand, requires modification to overcome the burden of $p E v o l v R$ expression in minimal media.

The great potential of OrthoRep and EvolvR lies in their high mutation rates, their scalability, and their compatibility with automation. A major limitation of classical, i.e. discontinuous, directed evolution is that, although a particular suite of mutations may define a fitness peak, the intermediate steps needed to sequentially reach that peak may not survive under strict selection. Automation of OrthoRep can facilitate dynamic selection regimes, as already demonstrated with the realtime/feedback-controlled culture device eVOLVER [29]. By tuning selection stringency, such regimes can help cross fitness valleys [24,25,29]. Moreover, the high mutation rates achievable in OrthoRep and EvolvR favor the accumulation of multiple mutations in a single replication step, which can help skip deleterious intermediate stages as said in the Introduction.

Continuous directed evolution in microbial platform organisms is a TINA ('There Is No Alternative') technology as far as plant enzyme improvement is concerned. Implementing directed enzyme evolution in plants is precluded by their large size, long life cycles, and metabolic complexity, which make it infeasible to generate billion-mutant populations and subject them to selection. Taking an enzyme out of the plant, evolving it in a microbe, and reinstalling it in the plant via genome editing is the only practical option. Such a strategy is potentially applicable to five broad areas of plant SynBio, as outlined below and in Figure 6.

(i) Improving kinetic properties. Directed evolution can outdo rational design by introducing multiple, potentially beneficial changes and going beyond known or predictable key residues [21]. Because it is carried out in vivo, continuous directed evolution has the further advantage of improving enzyme 
function under realistic conditions (e.g. substrate and product levels, macromolecular crowding), which in vitro screening protocols may mimic poorly [1].

(ii) Altering feedback inhibition. Many enzymes at the head of pathways are inhibited by downstream pathway intermediates or end-products and relieving this feedback inhibition is often effective in increasing flux in such pathways [49,50]. Selecting for feedback-insensitive microbial enzymes has a long track record of success [51-53] and can be extended to plant enzymes in microbes engineered to express them $[54,55]$.

(iii) Conferring herbicide resistance. Many herbicides act by inhibiting essential metabolic enzymes, and mutations that alleviate this inhibition confer resistance [56,57]. Continuous directed evolution has already been used to evolve antibiotic resistance in microbial enzymes [26,27]; this approach could be similarly applied to evolve herbicide-resistant crop enzymes. Using genome editing to put the resistant enzyme back in the crop would avoid using transgenes, which are now banned in various countries [58].

(iv) Creating new-to-nature functions. Because continuous directed evolution dramatically improves access to the vast protein design landscape, it can create new-to-nature features faster than classic directed evolution [22,59]. Such new features include altered substrate specificity [60] and catalysis of a different type of reaction [8].

(v) Extending enzyme life. The unnecessarily short working lives of certain plant enzymes wastes resources [3]. Short life is likely due in part to damage to active site residues by chemically reactive substrates or products or by catalytic misfires [61]. A recently introduced metric for enzyme working

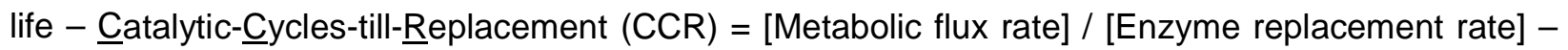
helps identify enzymes that are candidates for life-lengthening [61]. Continuous directed evolution could potentially be applied to increase enzyme longevity in vivo, i.e. to raise CCR, e.g. by driving replacement of damage-prone residues within the active site by less vulnerable alternatives.

\section{AUTHOR CONTRIBUTIONS}

J.D.G. and J.J. performed the experiments, J.J., C.C.R and A.D.H. designed the research. L.T.-R and A.A.J provided experimental support and were supervised by C.R.R. and C.C.L. respectively. A.D.H conceptualized the project and supervised J.D.G., J.J. and J.A.P. J.D.G, J.J., and J.A.P. wrote the initial draft under the supervision of A.D.H. and all authors contributed to review and editing. 


\section{FUNDING}

This research was supported by U.S. Department of Energy award DE-SC0020153 (to A.D.H. and C.R.R.) and by an endowment from the C.V. Griffin Sr. Foundation.

\section{REFERENCES}

1. Pettersson, G. (1989) Effect of evolution on the kinetic properties of enzymes. Eur. J. Biochem. 184, 561-566.

2. Bar-Even, A., Noor, E., Savir, Y., Liebermeister, W., Davidi, D. et al. (2011) The moderately efficient enzyme: evolutionary and physicochemical trends shaping enzyme parameters. Biochemistry 50, 4402-4410.

3. Amthor, J. S., Bar-Even, A., Hanson, A. D., Harvey Millar, A., Stitt, M., Sweetlove, L. J., Tyerman, S. D. (2019) Engineering strategies to boost crop productivity by cutting respiratory carbon loss. Plant Cell 31, 297-314.

4. Stitt, M. (2013) Progress in understanding and engineering primary plant metabolism. Curr. Opin. Biotechnol. 24, 229-238.

5. Jez, J. M., Lee, S. S. G., and Sherp, A. M. (2016) The next green movement: plant biology for the environment and sustainability. Science 353, 1241-1244.

6. Stewart Jr., C. N., Patron, N., Hanson, A. D., and Jez, J. M. (2018) Plant metabolic engineering in the synthetic biology era: Plant chassis selection. Plant Cell Rep. 37, 13571358.

7. Wurtzel, E. T., Vickers, C. E., Hanson, A. D., Millar, A. H., Cooper, M., et al. (2019) Revolutionizing agriculture with synthetic biology. Nat. Plants 5, 1207-1210.

8. Jeschek, M., Reuter, R., Heinisch, T., Trindler, C., Kleur, J., et al. (2016) Directed evolution of artificial metalloenzymes for in vivo metathesis. Nature. 537, 661-665.

9. Trudeau, D. L., Edlich-Muth, C., Zarzycki, J., Scheffen, M., Goldsmith, M. et at. (2018) Design and in vitro realization of carbon-conserving photorespiration. Proc. Natl. Acad. Sci. USA 115, E11455-E11464.

10. Engqvist, M. K. M. and Rabe, K. S. (2019) Applications of protein engineering and directed evolution in plant research. Plant Physiol. 179, 907-917.

11. Peracchi, A. (2001) Enzyme catalysis: removing chemically "essential" residues by sitedirected mutagenesis. Trends Biochem. Sci. 26, 497-503.

12. Spreitzer, R. J. and Salvucci, M. E. (2002) Rubisco: structure, regulatory interactions, and possibilities for a better enzyme. Annu. Rev. Plant Biol. 53, 449-475.

13. Nishitani, Y., Yoshida, S., Fujihashi, M., Kitagawa, K., Doi, T. et al. (2010) Structure-based catalytic optimization of a type III Rubisco from a hyperthermophile. J. Biol. Chem. 285, 39339-39347.

14. Ott, C. M., Smith, B. D., Portis, A. R. Jr., and Spreitzer, R. J. (2000) Activase region on chloroplast ribulose-1,5-bisphosphate carboxylase/oxygenase: nonconservative substitution in the large subunit alters species specificity of protein interaction. J. Biol. Chem. 275, 26241-44.

15. Larson, E. M., O’Brien, C. M., Zhu, G., Spreitzer, R. J., and Portis, A. R. Jr. (1997) Specificity for activase is changed by a Pro-89 to Arg substitution in the large subunit of ribulose-1,5bisphosphate carboxylase oxygenase. J. Biol. Chem. 272, 17033-37.

16. Yokota, A., Higashioka, M., and Wadano, A. (1992) Regulation of the activity of ribulose-1,5bisphosphate carboxylase/oxygenase through cooperative binding of 6-phosphogluconate to its regulatory sites. Eur. J. Biochem. 208, 547-557.

17. Parry, M. A. J., Keys, A. J., Madgwick, P. J., Carmo-Silva, A. E., and Andralojc, P. J. (2008) Rubisco regulation: a role for inhibitors, J. Exp. Bot. 59, 1569-1580.

18. Silver, P. A., Way, J. C., Arnold, F. H., Meyerowitz, J. T. (2014) Synthetic biology: Engineering explored. Nature 509, 166-167. 
19. Zeymer, C. and Hilvert, D. (2018) Directed evolution of protein catalysis. Annu. Rev. Biochem. 87, 131-157.

20. Lobkovsky, A. E., Wolf, Y. I., and Koonin, E. V. (2011) Predictability of evolutionary trajectories in fitness landscapes. PLoS Comput. Biol. 7: e1002302.

21. Goldsmith, M. and Tawfik, D. S. (2017) Enzyme engineering: reaching the maximal catalytic efficiency peak. Curr. Opin. Struct. Biol. 47, 140-150.

22. Zhong, Z. and Liu, C. (2019) Probing pathways of adapting with continuous evolution. Curr. Opin. Syst. Biol. 14, 18-24.

23. Esvelt, K. M., Carlson, J. C., and Liu, D. R. (2011) A system for the continuous directed evolution of biomolecules. Nature 472, 499-503.

24. Dickinson, B., Packer, M., Badran, A., and Liu, D. R. (2014) A system for the continuous directed evolution of proteases rapidly reveals drug-resistance mutations. Nat. Commun. 5, 5352.

25. Morrison, M. S., Podracky, C. J., and Liu D. R. (2020) The developing toolkit of continuous directed evolution. Nat. Chem. Biol. 16, 610-619.

26. Ravikumar, A., Arzumanyan, G. A., Obadi, M. K. A., Javanpour, A. A., and Liu, C. C. (2018) Scalable, continuous evolution of genes at mutation rates above genomic error thresholds. Cell 175, 1946-1957.

27. Halperin, S. O., Tou, C. J., Wong, E. B., Modavi, C., Schaffer, D. V. et al. (2018) CRISPRguide DNA polymerases enable diversification of all nucleotides in a tunable window. Nature 560, 248-252.

28. Álvarez, B., Mencía, M., de Lorenzo, V., and Fernández, L. A. (2020) In vivo diversification of target genomic using processive T7 RNA polymerase-base deaminase fusions blocked by RNA-guided dCas9. BioRxiv 850974

29. Zhong, Z., Wong B. G., Ravikumar, A., Arzumanyan, A., Khalil, A.S. et al. (2020). Automated continuous evolution of proteins in vivo. ACS Synth. Biol. 9, 1270-1276.

30. Gunge, N. and Sakaguchi, K. (1981) Intergeneric transfer of deoxyribonucleic acid killer plasmids, pGKI1 and pGKI2, from Kluyveromyces lactis into Saccharomyces cerevisiae by cell fusion. J. Bacteriol. 147, 155-160.

31. Kämper, J., Esser, K., Gunge, N., and Meinhardt, F. (1991) Heterologous gene expression on the linear DNA killer plasmid from Kluyveromyces lactis. Curr. Genet. 19, 109-118.

32. Ravikumar, A., Arrieta, A., and Liu, C. C. (2014) An orthogonal DNA replication system in yeast. Nat. Chem. Biol. 10, 175-177.

33. Chatterjee, A., Abeydeera, N. D., Bale, S., Pai, P. J., Dorrestein, P. C. et al. (2011) Saccharomyces cerevisiae Thi4p is a suicide thiamine thiazole synthase. Nature 478, 542546.

34. Hanson, A. D., Amthor, J. S., Sun, J., Niehaus, T. D., Gregory III, J. F. et al. (2018) Redesigning thiamin synthesis: Prospects and potential payoffs. Plant Sci. 273, 92-99.

35. Joshi J., Beaudoin, G. A., Patterson, J. A., García-García, J. D., Belisle, E., et al. (2020) Bioinformatic and experimental evidence for suicidal and catalytic plant THI4s. Biochem. J. 477, 2055-2069.

36. Zhang, X., Eser, B. E., Chanani, P. K., Begley, T. P., Ealick, S. E. (2016) Structural basis for iron-mediated sulfur transfer in archael and yeast thiazole synthases. Biochemistry 55, 18261838.

37. Eser, B. E., Zhang, X., Chanani, P. K., Begley, T. P., Ealick, S. E. (2016) From suicide enzyme to catalyst: the iron-dependent sulfide transfer in Methanococcus jannaschii thiazole biosynthesis. J. Am. Chem. Soc. 138, 3639-3642.

38. Sun, J., Singler, C. L., Beaudoin, G. A. W., Joshi, J.; Patterson, J. A. et al. (2019) Partsprospecting for a high-efficiency thiamin thiazole biosynthesis pathway. Plant Physiol. 179, 958-968.

39. Zhong, Z., Ravikumar, A., and Liu, C. C. (2018) Tunable expression systems for orthogonal DNA replication. ACS Synth. Biol. 7, 2930-2934. 
40. Mount, R. C., Jordan, B. E., and Hadfield, C. (1996) Transformation of lithium-treated yeast cells and the selection of auxotrophic and dominant markers. Methods Mol. Biol. 53, 139145.

41. Curran, B. P. and Bugeja, V. C. (1996) Protoplast fusion in Saccharomyces cerevisiae. Methods Mol. Biol. 53, 45-9.

42. Javanpour, A. and Liu, C. (2019) Genetic compatibility and extensibility of orthogonal replication. ACS Synth. Biol. 8, 1249-1256.

43. Wang, Y., Zhong, Y., Zhou, Y., Tanaseichuk, O., Li, L. et al. (2019) Identification of a Xist silencing domain by Tiling CRISPR. Sci. Rep. 9, 2408.

44. Lutz, R., and Bujard, H. (1997) Independent and tight regulation of transcriptional units in Escherichia coli via the $\mathrm{LacR} / \mathrm{O}$, the TetR/O and AraC/I1-I2 regulatory elements. Nucleic Acids Res. 25, 1203-1210

45. Stewart, V. and Parales, J. (1988) Identification and expression of genes narL and narX of the nar (nitrate reductase) locus in Escherichia coli K-12. J. Bacteriol. 170, 1589-1597.

46. Korshunov, S., Imlay, K.R., and Imlay, J.A. (2016) The cytochrome bd oxidase of Escherichia coli prevents respiratory inhibition by endogenous and exogenous hydrogen sulfide. Mol. Microbiol. 101, 62-77.

47. Paerl, R. W., Bertrand, E. M., Rowland, E., Schatt, P., Mehiri, M., et al. (2018) Carboxythiazole is a key microbial nutrient currency and critical component of thiamin biosynthesis. Sci. Rep. 8, 5940.

48. Nikel, P. I. and de Lorenzo, V. (2018) Pseudomonas putida as a functional chassis for industrial biocatalysis: from native biochemistry to trans-metabolism. Metab. Eng. 50, 142155.

49. Hong, Z., Lakkineni, K., Zhang, Z., and Verma, D.P. (2000) Removal of feedback inhibition of delta(1)-pyrroline-5-carboxylate synthase results in increasing proline accumulation and protection of plants from osmotic stress. Plant Physiol. 122, 1129-1136.

50. Whitcomb, S. J., Rakpenthai, A., Brückner, F., Fischer, A., Parmar, S., et al. (2020) Cysteine and methionine biosynthetic enzymes have distinct effects on seed nutritional quality and on molecular phenotypes associate with accumulation of a methionine-rich seed storage protein in rice. Front. Plant Sci. 11, 1118.

51. Cohen, G. N., Patte, J. C., and Truffa-Bachi, P. (1965) Parallel modifications caused by mutations in two enzymes concerned with the biosynthesis of threonine in Escherichia coli. Biochem. Biophys. Res. Commun. 19, 546-550.

52. Báez-Viveros, J. L., Osuna, J., Hernández-Chávez, G., Soberón, X., Bolívar, F., et al. (2004) Metabolic engineering and protein directed evolution increasing the yield of $L$-phenylalanine synthesized from glucose in Escherichia coli. Biotechnol. Bioeneg. 87, 516-524.

53. Petit, C., Kim, Y., Lee, S., Brown, J., Larsen, E. et al. (2018) Reduction of feedback inhibition in homoserine kinase (ThrB) of Corynebacterium glutamicum enhances I-threonine biosynthesis. ACS Omega 3, 1178-1186.

54. Alberstein, M., Eisenstein, M., and Abeliovich, H. (2012) Removing allosteric feedback inhibition of tomato 4-coumarate:CoA ligase by directed evolution. Plant J. 69, 57-69.

55. Ning, J., Moghe, G. D., Leong, B., Kim, J., Ofner, I. et al. (2015) A feedback-insensitive isopropylmalate synthase affects acylsugar composition in cultivated and wild tomato. Plant Physiol. 169, 1821-1835.

56. Rangani, G., Salas-Perez, R. A., Aponte, R. A., Knapp, M., Craig, I. R. et al. (2019) A novel single-site mutation in the catalytic domain of protoporphyrinogen oxidase IX (PPO) confers resistance to PPO-inhibiting herbicides. Front. Plant Sci. 15, 568.

57. Hawkes, T. R., Langford, M. P., Viner, R., Blain, R. E., Callaghan, F. M. et al. (2019) Characterization of 4-hydroxyphenylpyruvate dioxygenases, inhibition by herbicides and engineering for herbicide tolerance in crops. Pestic. Biochem. Physiol. 156, 9-28.

58. Mackelprang, R. and Lemaux, P. G. (2020) Genetic engineering and editing of plants: an analysis of new and persisting questions. Annu. Rev. Plant Biol. 71, 659-587. 
59. Badran, A. H. and Liu, D. R. (2015) In vivo continuous directed evolution. Curr. Opin. Chem. Biol. 24, 1-10.

60. Williams, J. G., Domann, S., Nelson, A., and Berry, A. (2003) Modifying the stereochemistry of an enzyme-catalyzed reaction by directed evolution. Proc. Natl. Acad. Sci. USA 100, 3143-3148.

61. Tivendale, N. D., Hanson, A. D., Henry, C. S., Hegeman, A. D., and Millar, A.H. (2020) Enzymes as parts in need of replacement - and how to extend their working life. Trends Plant Sci. 25, 661-669. 


\section{FIGURES}

Figure 1. Rugged fitness landscape with multiple fitness peaks. The $x$ and $y$ axes represent the multidimensional sequence space defined by different possible sites and identities of mutations in an enzyme; the $z$-axis is fitness as a function of sequence. Red arrows show possible evolutionary paths created by mutation and selection. An enzyme at suboptimal fitness level A could potentially reach local fitness peak $B$ or global optimal peak $C$. Because the path from $A$ to $C$ passes through a fitness valley it is more difficult to follow under selection than the continuously upward path from $A$ to B.

\section{Figure 2. The OrthoRep and EvolvR continuous directed evolution systems.}

Schematic overviews of the two continuous evolution systems evaluated in this study. (A) The yeast OrthoRep system consists of an error-prone DNA polymerase, encoded by a nuclear plasmid, that replicates cytoplasmic linear plasmid $p 1$, and only $p 1$. The $p 1$ plasmid is engineered to express the target gene. Cytoplasmic linear plasmid p2 carries the machinery needed to transcribe the genes on both $\mathrm{p} 1$ and $\mathrm{p} 2$, and a high-fidelity DNA polymerase that replicates p2 alone. (B) The E. coli EvolvR system comprises the pEvolvR plasmid, encoding an nCas9 nickase-error-prone DNA polymerase I fusion plus a guide RNA, and pTarget encoding the target gene.

Figure 3. Construction of the multipurpose p1 integration vector for OrthoRep.

(A) A linker sequence containing a Smal site was introduced into the Sphl site of the GR306 plasmid in such a way as to retain the Sphl site. This linker allowed insertion of the poly-A/RZ module from 172-YTK-P4 into GR306. The resulting construct is a multipurpose integration vector from which the resident $m$ Kate gene can be excised with $\mathrm{Nsil}$ and Sphl and replaced with a target gene. (B) The yeast THI4 (ScTHI4) gene was introduced into the multipurpose integration vector as above; the construct was linearized by Scal and transformed into yeast strain GA-Y319, and homologous recombination integrated ScTHI4 into the p1 plasmid. Finally, protoplast fusions were carried out between GA-Y319 (donor strain) and $\triangle T H I 4$ BY4741 strains carrying the DNA polymerases TP_DNAP1 wt, TP_DNAP1 611, or TP_DNAP1 633 (receptor strains). Selective medium without leucine, histidine, and tryptophan favored the growth of $\triangle T H I 4$ BY4741 cells carrying the full OrthoRep system plus ScTHI4.

Figure 4. Complementation of a yeast $\Delta T H I 4$ strain by the yeast $T H I 4$ gene encoded on the OrthoRep p1 plasmid.

(A) Three clones of each of the OrthoRep-containing $\Delta T H I 4$ strains recovered from protoplast fusions (see Figure 3) were grown on agar plates containing SC minus leucine, histidine, and 
tryptophan, and supplemented with $2 \%$ (w/v) glucose, $1 \mathrm{~g} / \mathrm{L}$ glutamate and $200 \mu \mathrm{g} / \mathrm{mL}$ G418, in absence or presence of $100 \mathrm{nM}$ thiamin. Clones were collected in $20 \mu \mathrm{L}$ of the same selective medium (with agar, thiamin, and G418 omitted), from which $3 \mu \mathrm{L}$ was used to streak fresh plates. Plates containing cells carrying TP_DNAP1 wt, TP_DNAP1 611, and TP_DNAP1 633, were imaged after $4.5,7$, and 8 days at $30^{\circ} \mathrm{C}$, respectively. (B) Independent clones from the thiamin-containing plates were used to assess complementation in liquid medium (composition as above, minus agar). Growth of strains containing p1_empty or p1_ScTHI4, with or without 100 nM thiamin was measured at $\mathrm{OD}_{660 \mathrm{~nm}}$. Values are means \pm standard error (SE) of independent replicates (three for TP_DNAP1 611 strains and seven for TP_DNAP1 wt strains). Where no error bars appear, they are smaller than the symbol.

Figure 5. Attempted complementation of an E. coli $\Delta$ thiG strain by TaTHI4 using components of the EvolvR system.

E. coli $\Delta$ thiG cells harboring the pCDFDuet-placO vector alone (pEv) or containing the TaTHI4 (pTaTH/4) gene were cultured in MOPS minimal medium containing $0.2 \%(\mathrm{w} / \mathrm{v})$ glycerol, $1 \mathrm{mM}$ IPTG, and $1 \mathrm{mM}$ cysteine with $100 \mathrm{nM}$ thiamin (A) or without thiamin (C). $\Delta$ thiG cells harboring both pEvolvR and pEv or pTaTHI4 were cultured in MOPS minimal medium plus glycerol, cysteine, and IPTG as above, with $100 \mathrm{nM}$ thiamin (B) or without thiamin (D). Values are means \pm standard error (SE) of three independent replicates. Where no error bars appear, they are smaller than the symbol.

Figure 6. Potential applications of continuous directed evolution in plant SynBio. 
bioRxiv preprint doi: https://doi.org/10.1101/2020 08.26.265678; this version posted August 27, 2020. The copyright holder for this preprint (which was not certified by peer review) is the author/funder, who has granted bioRxiv a license to display the preprint in perpetuity. It is made available under aCC-BY-NC-ND 4.0 International license.

\section{Figure 1}

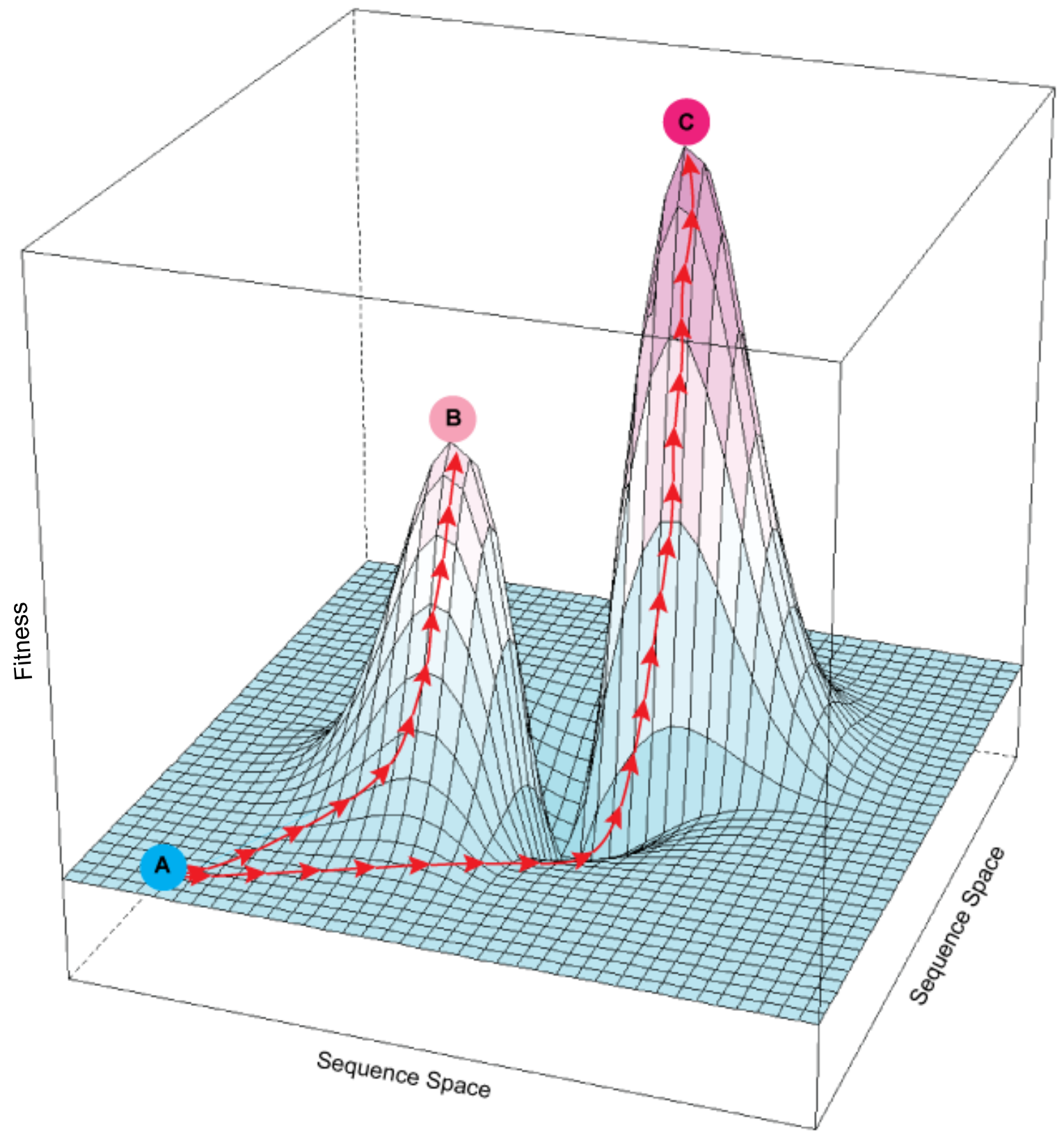


bioRxiv preprint doi: https://doi.org/10.1101/2020.08.26.265678; this version posted August 27, 2020. The copyright holder for this preprint (which was not certified by peer review) is the author/funder, who has granted bioRxiv a license to display the preprint in perpetuity. It is made available under aCC-BY-NC-ND 4.0 International license.

\section{Figure 2}

A

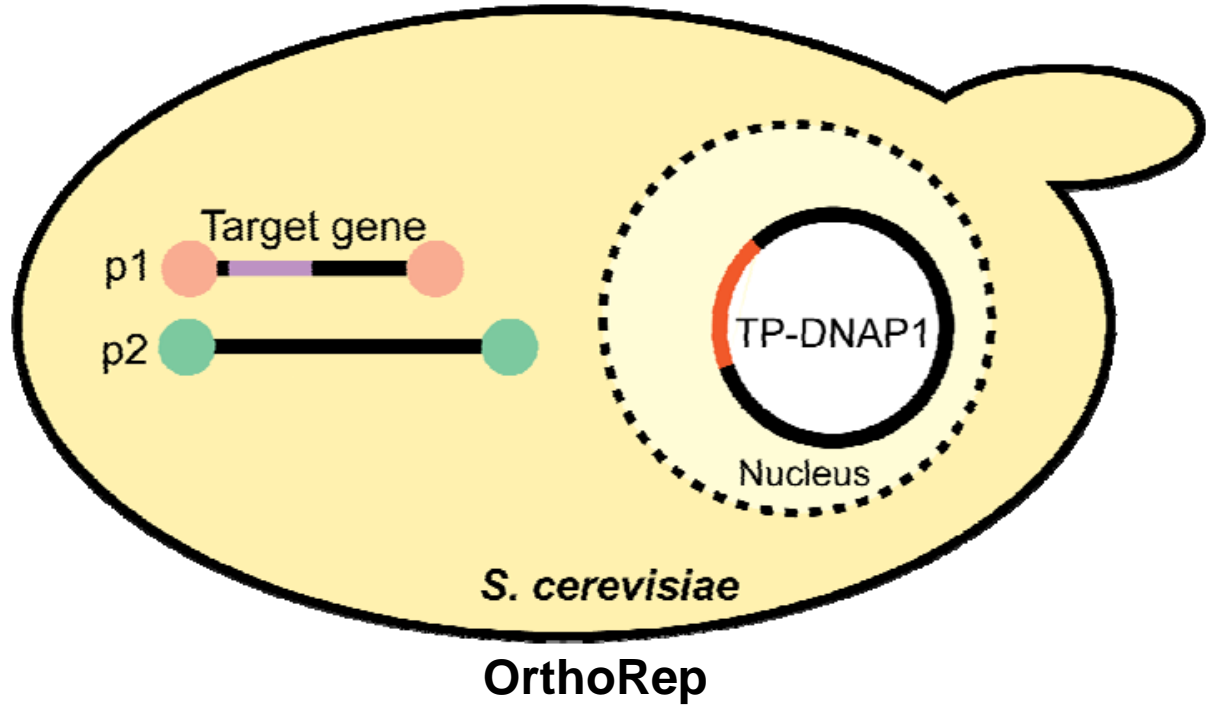

B

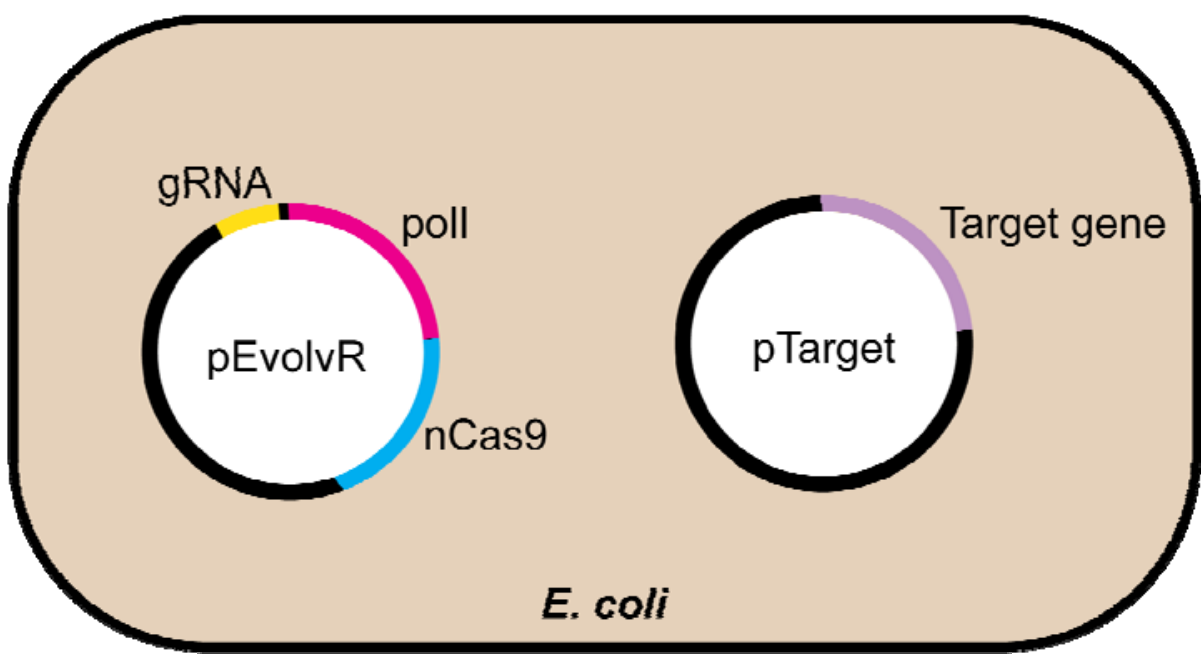

EvolvR 
bioRxiv preprint doi: https://doi.org/10.1101/2020.08 26.265678. this version posted August 27, 2020. The copyright holder for this preprint (which was not certified by peer review) is the author/funder, who has granted bioRxiv a license to display the preprint in perpetuity. It is made available under aCC-BY-NC-ND 4.0 International license.

\section{Figure 3}

A

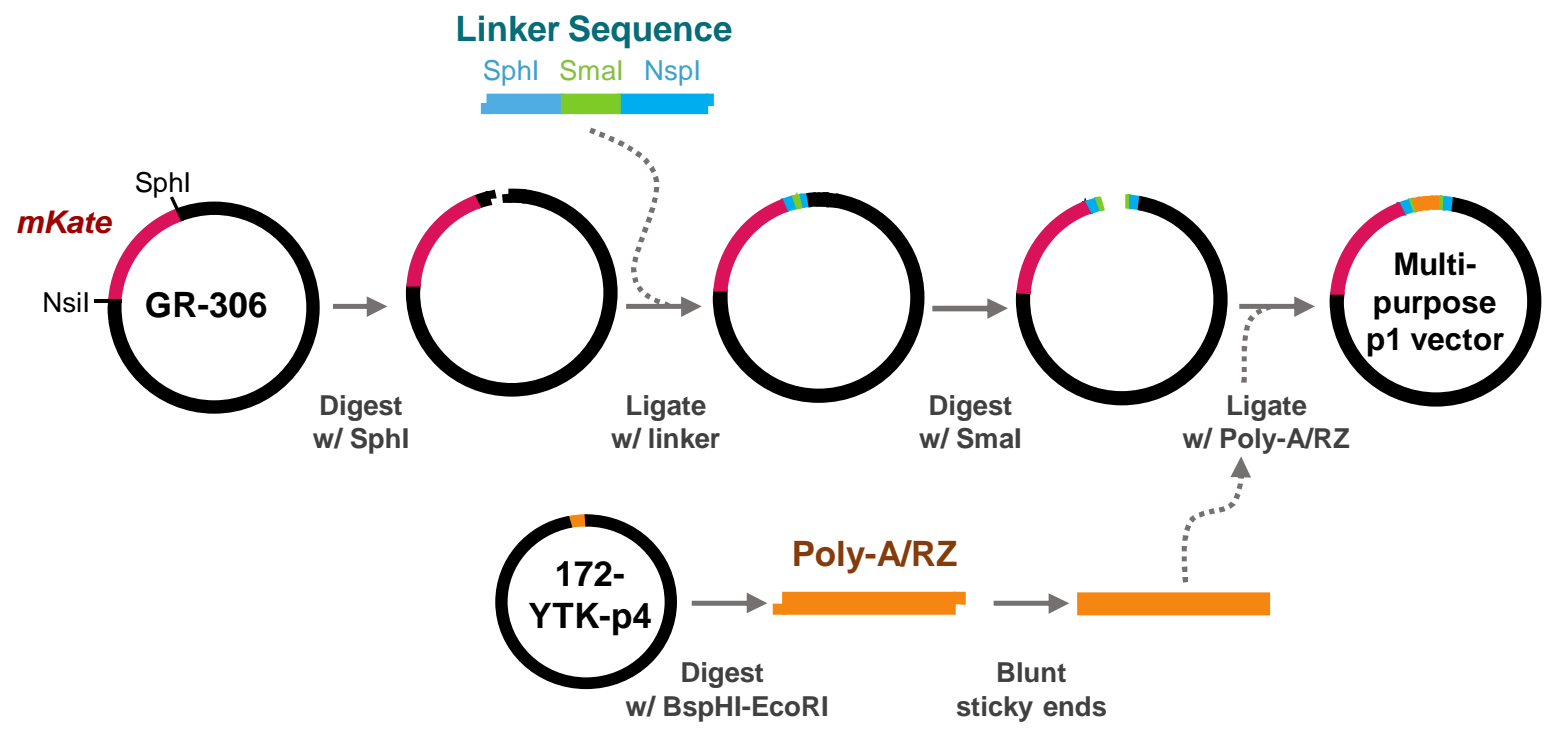

B

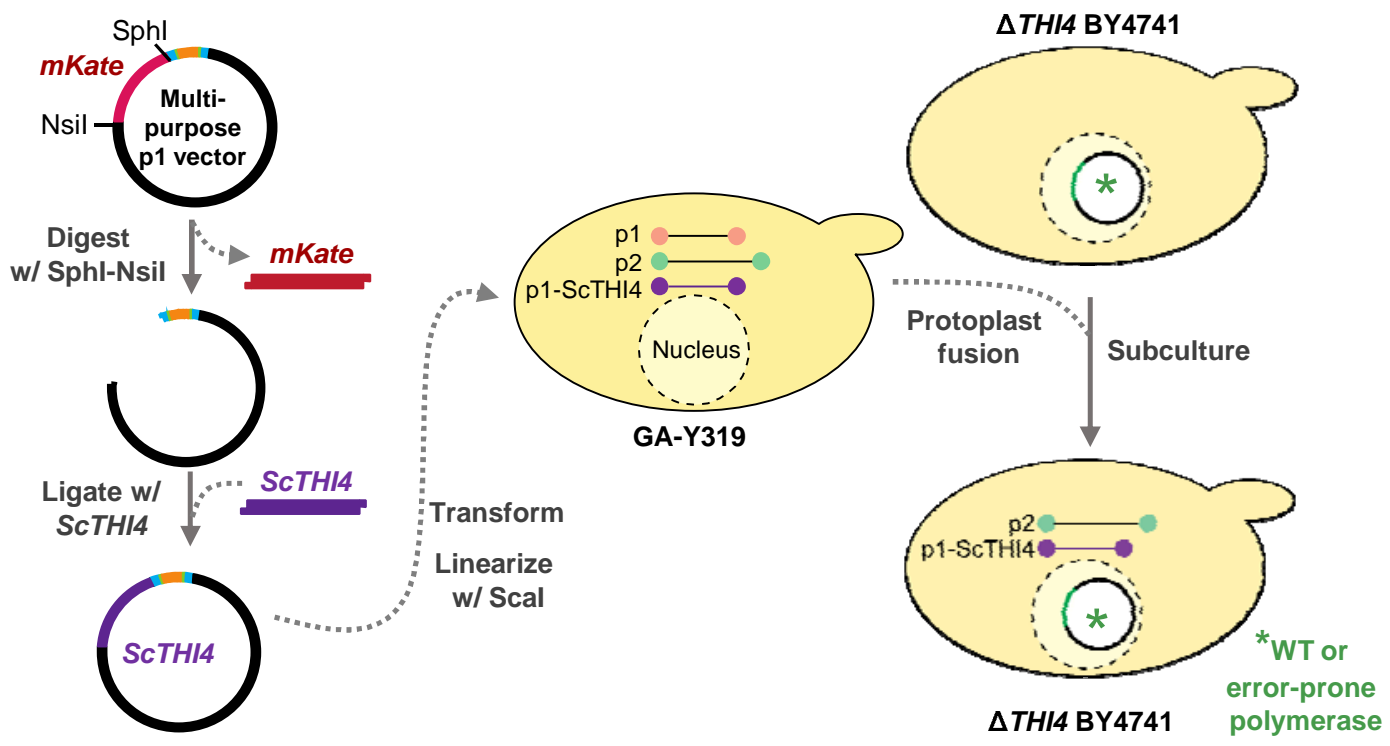




\section{Figure 4}

A

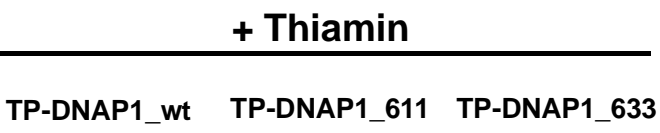

p1_empty
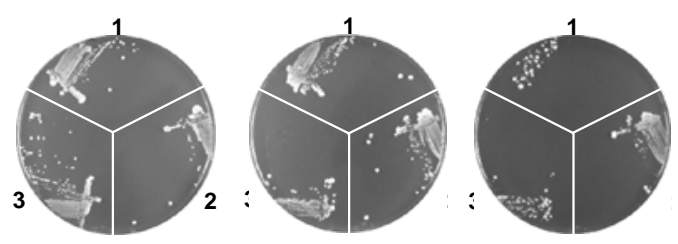

p1_ScThi4
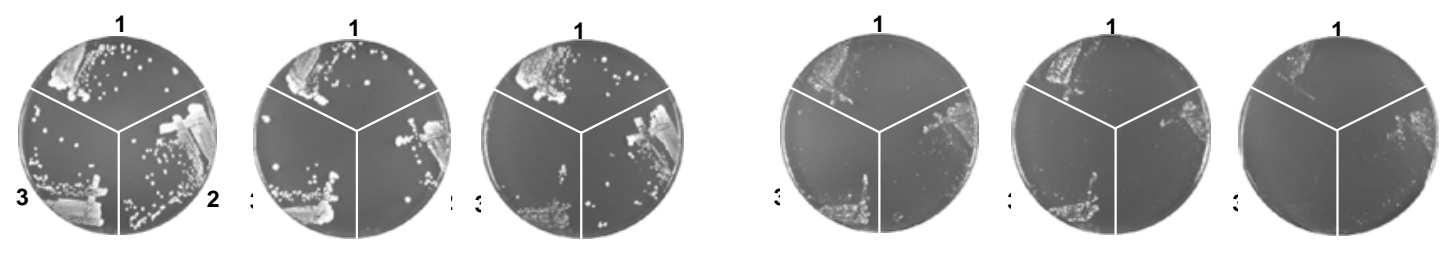

B
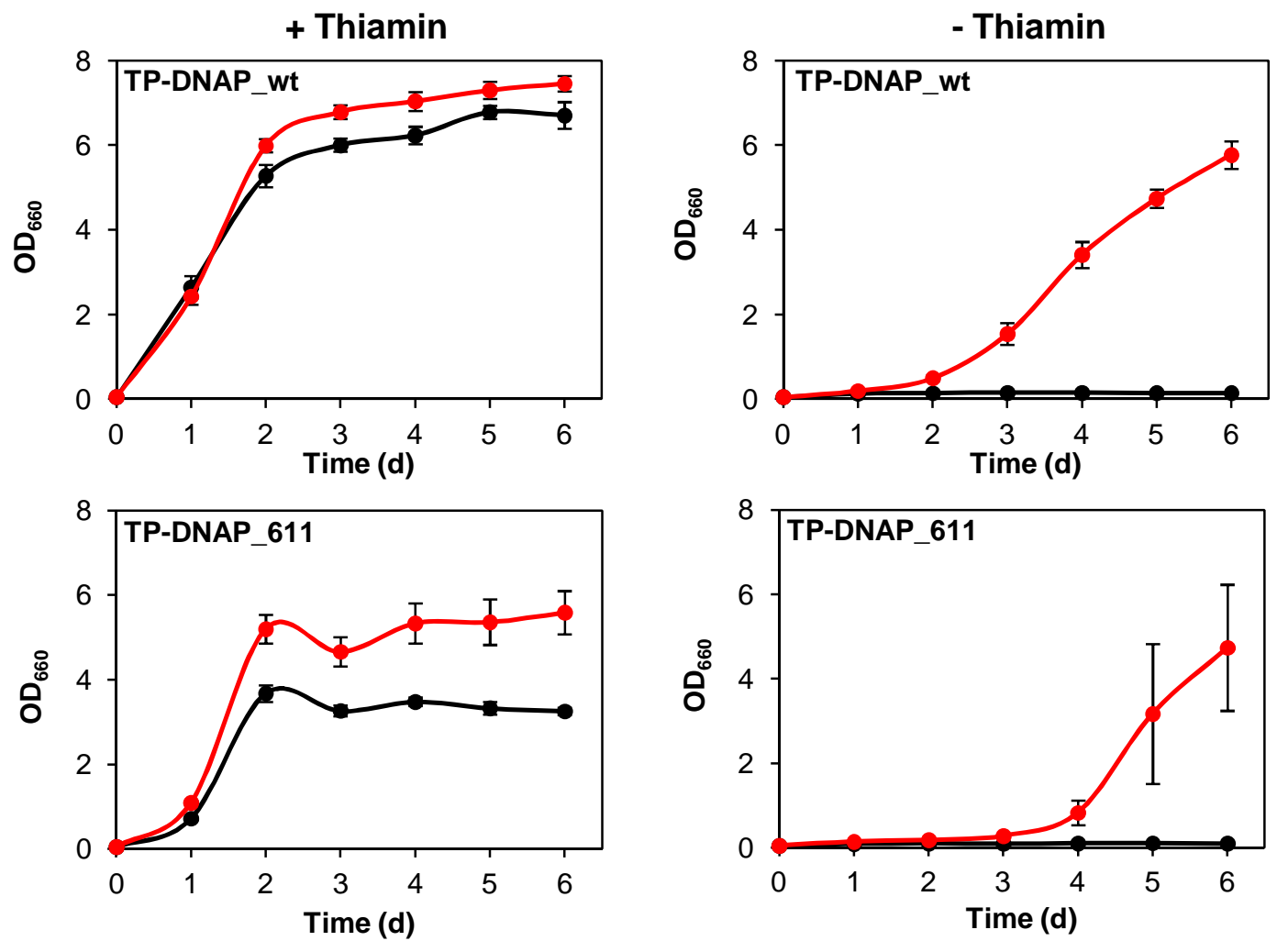

$\multimap$ p1_empty

- Thiamin
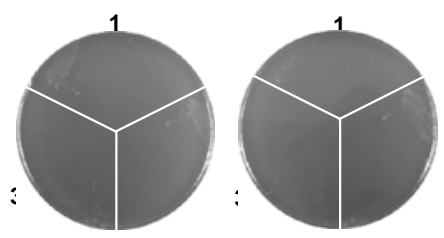

TP-DNAP1_wt TP-DNAP1 611 TP-DNAP1 633

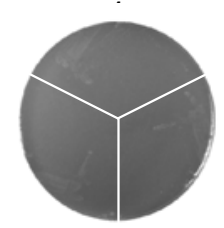




\section{Figure 5}

A

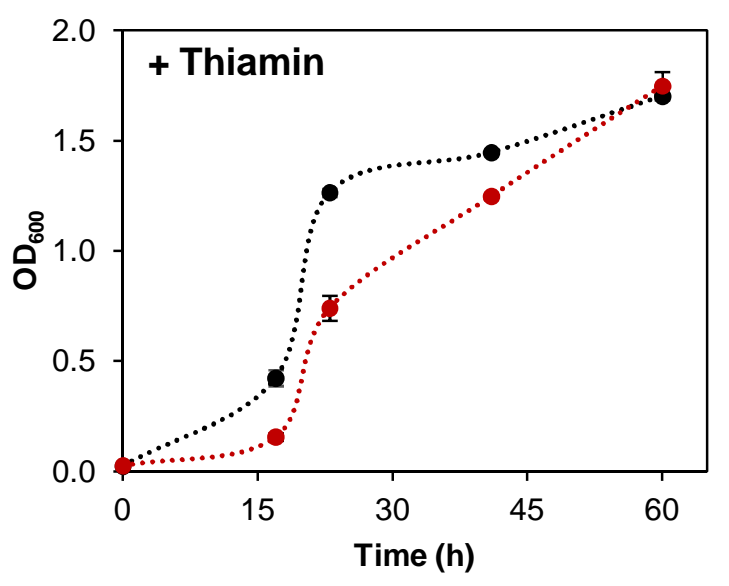

……pEv $\quad \cdots \cdot \cdots \cdot$ pTaTHI4

C

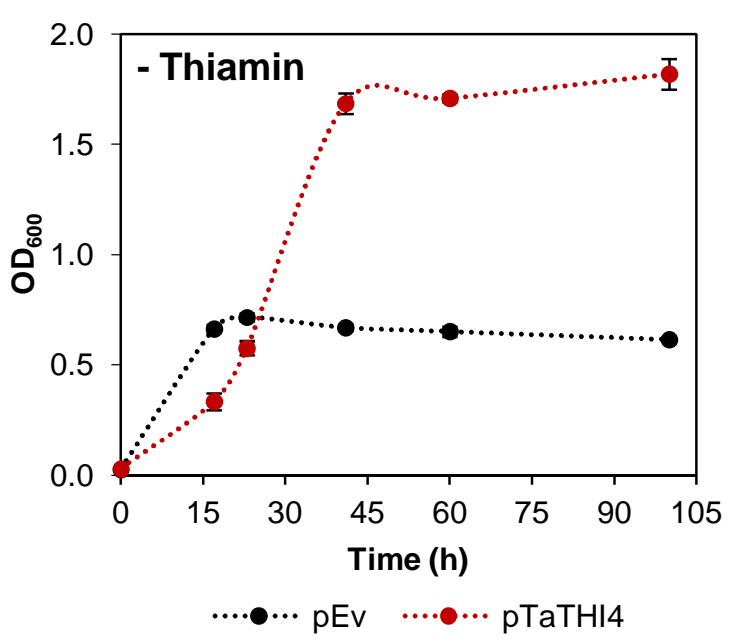

B

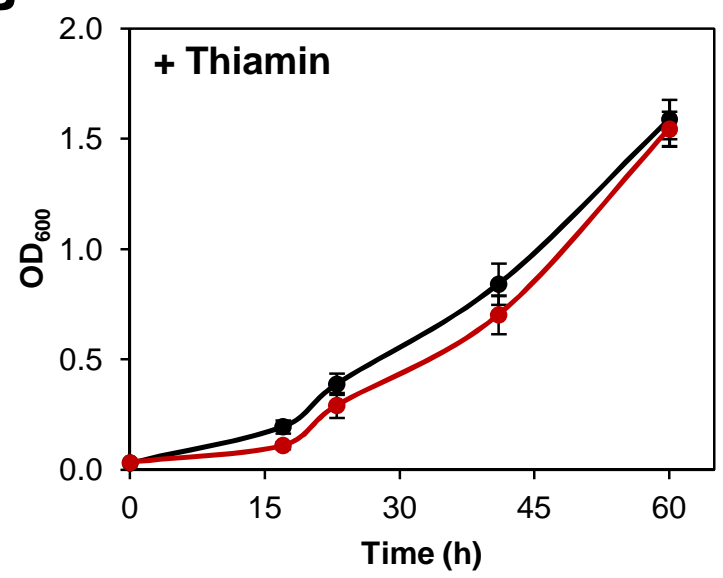

$\rightarrow p E v+E v o l v R \rightarrow p T a T H I 4+E v o l v R$

D

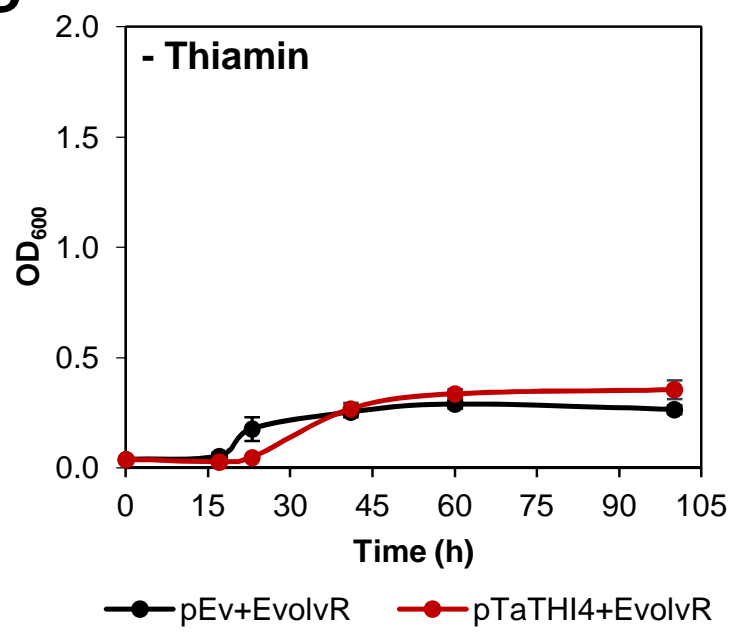


bioRxiv preprint doi: https://doi.org/10.1101/2020.08.26.265678; this version posted August 27, 2020. The copyright holder for this preprint (which was not certified by peer review) is the author/funder, who has granted bioRxiv a license to display the preprint in perpetuity. It is made available under aCC-BY-NC-ND 4.0 International license.

\section{Figure 6}

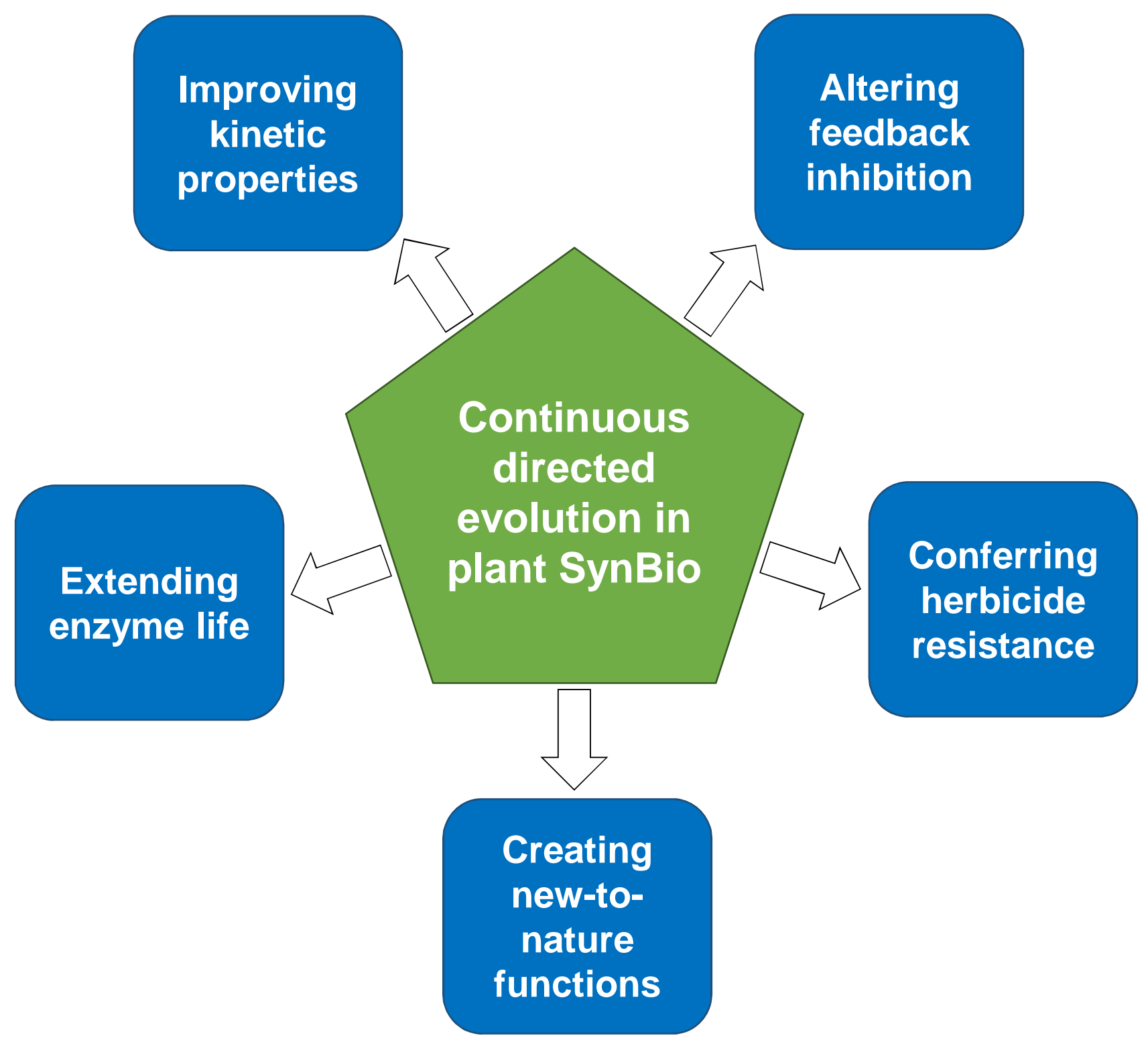

